\title{
Use of Laryngeal Mask Airway in Total Thyroidectomy in a Patient with Ocular Myasthenia and Graves’ Disease
}

\author{
Samantha Shilpage ${ }^{1}$, Ranil Fernando ${ }^{2}$, Nalinda Munasinghe ${ }^{3}$, Bhagya Gunathilaka ${ }^{4}$
}

\begin{abstract}
Background: The association between Graves' disease (GD) and ocular myasthenia (OM) is well known. Total thyroidectomy gives a permanent "cure" for GD and the literature reports a varying progression of myasthenia gravis (MG) in such patients after surgery. The main issue in patients with MG is the difficulties with the use of muscle relaxants during anesthesia. In the ensuing case report, a patient with OM and GD who had a total thyroidectomy under general anesthesia (GA) with laryngeal mask airway (LMA) without muscle relaxants is described.

Case report: A 40-year-old man was clinically and biochemically diagnosed to have GD. Subsequently, he developed bilateral ptosis which was diagnosed as OM by a neurologist. Though his OM responded to treatment, his GD was not well controlled. He was admitted, monitored, and made euthyroid by adjusting the carbimazole dose. A decision was made to undertake total thyroidectomy for GD, with total intravenous anesthesia and Prosea ${ }^{\oplus}$ LMA without muscle relaxants following a discussion with the anesthetic team. A conventional total thyroidectomy was performed using nerve encountering, parathyroid preserving, and capsular dissection technique. The patient made an uncomplicated recovery. Conclusion: A technique combining total intravenous anesthesia and a LMA without muscle relaxants for thyroid surgery in a patient with MG has advantages over a standard method. The use of LMA in other thyroid surgeries must be further evaluated for future use.

Clinical significance: Coexistence of MG and GD should be in mind when treating the patients with GD. Individualize the use of muscle relaxants in GA and best to avoid whenever possible.

Keywords: Conventional total thyroidectomy, Graves' disease, Laryngeal mask airway, Myasthenia gravis, Ocular myasthenia.

World Journal of Endocrine Surgery (2019): 10.5005/jp-journals-10002-1250
\end{abstract}

\section{INTRODUCTION}

The association between Graves' disease (GD) and ocular myasthenia $(\mathrm{OM})$ is well known. ${ }^{1}$ The exact incidence is yet to be ascertained.

Total thyroidectomy gives a permanent "cure" for GD and the literature reports a varying progression of myasthenia gravis (MG) in such patients after surgery. ${ }^{2}$

The main issue in patients with MG is the difficulties with the use of muscle relaxants during anesthesia. The duration of action and recovery of the patients is unpredictable.

In the ensuing case report, a patient with OM and GD who had a total thyroidectomy under general anesthesia (GA) with laryngeal mask airway (LMA) without muscle relaxants is described. This has not been reported previously in the literature.

\section{Case Report}

A 40-year-old man, who was clinically and biochemically hyperthyroid [FT3-23.68 (3.4-4.8) pmol/L, FT4-7.77 (10-28.3) $\mathrm{pmol} / \mathrm{L}$, and TSH-0.003 (0.4-4.6) $\mathrm{mlU} / \mathrm{L}]$, was diagnosed to have GD. He was treated with $20 \mathrm{mg}$ of carbimazole 8 hourly. Three months after commencement of antithyroid drugs, he developed bilateral ptosis (more on right side) which was diagnosed as "OM" by a neurologist. He was prescribed neostigmine ( $15 \mathrm{mg}, 8$ hourly) and the OM was well controlled.

However, his hyperthyroidism was not controlled even after 12 months of antithyroid drugs. He was admitted, and his medication was titrated. Euthyroid status was confirmed biochemically (FT3$3.42 \mathrm{pmol} / \mathrm{L}, \mathrm{FT} 4-1.6 \mathrm{pmol} / \mathrm{L}$, and TSH$-3.4 \mathrm{mlU} / \mathrm{L}$ ) after 3 months. A decision was made to undertake total thyroidectomy for GD.

The patient's management was discussed with the anesthetic team and a preoperative decision was made to do the surgery
${ }^{1}$ Department of Surgery, Professorial Unit, North Colombo Teaching Hospital, Ragama, Sri Lanka

${ }^{2}$ Department of Surgery, Faculty of Medicine, University of Kalaniya, Sri Lanka

${ }^{3}$ Department of Surgery, Faculty of Medicine, University of Kelaniya, Ragama, Sri Lanka

${ }^{4}$ Department of Anesthesiology, North Colombo Teaching Hospital, Ragama, Sri Lanka

Corresponding Author: Samantha Shilpage, Department of Surgery, Professorial Unit, North Colombo Teaching Hospital, Ragama, Sri Lanka, Phone: +94 0773283641, e-mail: ssamantha_g@yahoo.com

How to cite this article: Shilpage $S$, Fernando $R$, Munasinghe $N$, Gunathilaka B. Use of Laryngeal Mask Airway in Total Thyroidectomy in a Patient with Ocular Myasthenia and Graves' Disease. World J Endoc Surg 2019;11(1):26-27.

Source of support: Nil

Conflict of interest: None

under total intravenous anesthesia with a Proseal $^{\oplus}$ LMA without muscle relaxants.

A conventional total thyroidectomy was performed using nerve encountering, parathyroid preserving, and capsular dissection technique. The duration from induction to full recovery is 2 hours. Retraction of the strap muscles and access to thyroid gland were not a problem without muscle relaxants. The only difficulty was the need to reposition the LMA which was displaced due to manipulation of the thyroid gland and the trachea. The patient made a complete recovery and the postoperative period was uncomplicated. On the following day, he was discharged with thyroxin $100 \mu \mathrm{g} /$ day and neostigmine $15 \mathrm{mg} 8$ hourly.

(0) The Author(s). 2019 Open Access This article is distributed under the terms of the Creative Commons Attribution 4.0 International License (https://creativecommons. org/licenses/by-nc/4.0/), which permits unrestricted use, distribution, and non-commercial reproduction in any medium, provided you give appropriate credit to the original author(s) and the source, provide a link to the Creative Commons license, and indicate if changes were made. The Creative Commons Public Domain Dedication waiver (http://creativecommons.org/publicdomain/zero/1.0/) applies to the data made available in this article, unless otherwise stated. 


\section{Discussion}

The standard method of anesthesia in thyroidectomy includes the use of intravenous and volatile anesthetics, opioid analgesics, neuromuscular blocking agents, and endotracheal intubation. In patients with MG, the need for securing the airway using an endotracheal tube needs to be balanced with the unpredictable duration of action and recovery from the effects of muscle relaxants. Volatile anesthetic agents may potentiate the action of muscle relaxants. ${ }^{3,4}$ LMA in combination with total intravenous anesthesia was used to circumvent these issues.

Literature describes the use of the LMA instead of the endotracheal tube particularly in difficulties with intubation. ${ }^{5}$ The use of LMAs for thyroidectomy is well described.

Repositioning of the LMA displaced during the procedure was easily carried out without disturbing the surgical field due to the unique method of draping used by the surgical team.

\section{Conclusion}

An anesthetic technique combining total intravenous anesthesia and a LMA without muscle relaxants for thyroid surgery in a patient with MG has advantages over a standard method.
The use of LMA in other thyroid surgeries must be further evaluated for future use.

\section{Clinical Significance}

Coexistence of MG and GD should be in mind when treating the patients with GD. Individualize the use of muscle relaxants in GA and best to avoid whenever possible.

\section{References}

1. Chhabra S, Pruthvi BC. Ocular myasthenia gravis in a setting of thyrotoxicosis. Indian J Endocrinol Metab 2013;17:341-343. DOI: 10.4103/2230-8210.109666.

2. Ali AS, Akavaram NR. Neuromuscular disorders in thyrotoxicosis. Am Fam Physician 1980;22:97-102.

3. Michael FR, Lee A. Anesthetic implications of concurrent diseases. In: Ronald DM, ed. Miller's Anesthesia, 7th edn, Philadelphia: Churchill Livingstone, 2010; 1115: pp. 19103-2899.

4. Abel M, Eisenkraft JB, et al. Anesthetic Implications of Myasthenia Gravis. THE Mt Sinai J Med January/March 2002.

5. Chun B-J, Bae J-S, et al. A Prospective Randomized Controlled Trial of the Laryngeal Mask Airway Versus the Endotracheal Intubation in the Thyroid Surgery: Evaluation of Postoperative Voice, and Laryngopharyngeal Symptom. World J Surg 2015;39(7):1713-1720. DOI: 10.1007/s00268-015-2995-7. 